\title{
Article \\ Tectoridin Stimulates the Activity of Human Dermal Papilla Cells and Promotes Hair Shaft Elongation in Mouse Vibrissae Hair Follicle Culture
}

\author{
Gary Ka-Wing Yuen ${ }^{1,2}$, Bryan Siu-Yin Ho ${ }^{2}$, Lish Sheng-Ying Lin ${ }^{2}$, Tina Ting-Xia Dong ${ }^{1,2}$ \\ and Karl Wah-Keung Tsim 1,2,*
}

1 Shenzhen Key Laboratory of Edible and Medicinal Bioresources, HKUST Shenzhen Research Institute, Shenzhen 518057, China; kwgyuen@ust.hk (G.K.-W.Y.); botina@ust.hk (T.T.-X.D.)

2 Division of Life Science and Centre for Chinese Medicine, The Hong Kong University of Science and Technology, Hong Kong, China; hosyb2002@yahoo.com.hk (B.S.-Y.H.); lishlin@ust.hk (L.S.-Y.L.)

* Correspondence: botsim@ust.hk; Tel.: +86-(852)-2358-7332

\section{check for}

updates

Citation: Yuen, G.K.-W.; Ho, B.S.-Y.;

Lin, L.S.-Y.; Dong, T.T.-X.; Tsim,

K.W.-K. Tectoridin Stimulates the Activity of Human Dermal Papilla Cells and Promotes Hair Shaft

Elongation in Mouse Vibrissae Hair Follicle Culture. Molecules 2022, 27, 400. https://doi.org/10.3390/ molecules 27020400

Academic Editors: Sibao Chen and Saverio Bettuzzi

Received: 18 November 2021

Accepted: 5 January 2022

Published: 8 January 2022

Publisher's Note: MDPI stays neutral with regard to jurisdictional claims in published maps and institutional affiliations.

Copyright: (C) 2022 by the authors. Licensee MDPI, Basel, Switzerland. This article is an open access article distributed under the terms and conditions of the Creative Commons Attribution (CC BY) license (https:// creativecommons.org/licenses/by/ $4.0 /)$.

\begin{abstract}
To search hair growth-promoting herbal extract, a screening platform of having HEK293T fibroblast being transfected with pTOPFLASH DNA construct was developed over a thousand of herbal extracts and phytochemicals were screened. One of the hits was ethanolic extract of Rhizoma Belamcandae, the rhizome of Belamcanda chinensis (L.) DC. Tectoridin, an isoflavone from Rhizoma Belamcandae, was shown to be responsible for this activation of promoter construct, inducing the transcription of pTOPFLASH in the transfected fibroblasts in a dose-dependent manner. The blockage by DKK-1 suggested the action of tectoridin could be mediated by the Wnt receptor. The hair growth-promoting effects of tectoridin were illustrated in human follicular dermal papilla cells and mouse vibrissae organ cultures. In tectoridin-treated dermal papilla cultures, an activation of Wnt signaling was demonstrated by various indicative markers, including TCF/LEF1 transcriptional activity, nuclear translocation of $\beta$-catenin, expressions level of mRNAs encoding axin-related protein, (AXIN2), $\beta$-catenin, lymphoid enhancer-binding factor-1 (LEF-1), insulin-like growth factor 1 (IGF-1) and alkaline phosphatase (ALP). In addition, an increase of hair shaft elongation was observed in cultured mouse vibrissae upon the treatment of tectoridin. Tectoridin, as well as the herbal extract of Rhizoma Belamcandae, possesses hair promoting activity, which deserves further development.
\end{abstract}

Keywords: Belamcanda chinensis (L.) DC.; tectoridin; hair growth; alopecia

\section{Introduction}

Hair growth is a dynamic process being controlled by hair follicle in a cyclic manner. The growth of hair has several important functions in social interaction, psychological wellbeing, and regulating body temperature [1]. Hair follicle undergoes the cycles of growth and regression throughout the lifetime. The hair follicle cycle is mainly classified into three phases: (i) the active hair growth phase (anagen); (ii) the regression phase (catagen); and (iii) the resting phase (telogen) [2]. The control of hair growth cycle relies on a delicate balance between growth promoting and inhibiting signals. The Wnt/ $\beta$-catenin signaling is known to play a role in the developmental processes of hair [3], and the activation of Wnt/ $\beta$-catenin signaling triggers the growth of hair follicle, as well as to stimulate the expressions of genes at the anagen phase of hair cycle [4]. The hair growth-promoting signals have been identified, including the signaling of wingless (Wnt), fibroblast growth factor (FGF), vascular endothelial growth factor (VEGF), insulin-like growth factor 1 (IGF-1). In contrast, transforming growth factor $\beta$ (TGF- $\beta$ ) and bone morphogenetic protein (BMP) are known to inhibit hair growth or to drive hair follicle into regression [5]. Dermal papilla cell (DPC), a cluster of specialized mesenchymal cells located the base of hair follicle, plays an important role in regulating hair growth [6], which secretes different paracrine and 
autocrine factors to regulate the growth cycle of hair follicle, as well as various intracellular activities inside the follicles. DPC provides different signals of Wnt, R-spondin, FGF, and Noggin in activating the follicular bulge stem cell, and thereafter which initiate the anagen phase [7].

Alopecia is the condition of progressive loss of terminal hair on the scalp or anywhere on the body [8]. Alopecia can be mainly divided into two categories, scarring and nonscarring [9]. Scarring alopecia refers to permanent hair loss, and the hair follicles are irreversibly destroyed, e.g., lichen planopilaris and folliculitis decalvans. Non-scarring alopecia refers to hair loss due to the hair cycle being disrupted, as in cases of alopecia areata, telogen effluvium and androgenic alopecia. In non-scarring alopecia, hair follicles are preserved, and which can be reactivated for hair regrowth [10]. During embryonic and adult stages, the Wnt/ $\beta$-catenin signaling plays an important role in hair morphogenesis and cycling $[3,11]$. Wnt3a and Wnt7b containing macrophage-extracellular vesicles can increase the proliferation of DPC and enhance hair follicle growth in male mice in vivo [12,13]. Thus, activators of Wnt/ $\beta$-catenin signaling could be considered for the treatment of hair loss. Several herbal extracts and phytochemicals have been shown to promote hair growth through Wnt/ $\beta$-catenin signaling. For example, baicalin was shown to promote hair growth in mouse and DPC via Wnt/ $\beta$-catenin signaling [14]. A Wnt/ $\beta$-catenin activator (SM04554) has entered the clinical trial in treating androgenetic alopecia [15].

Tectoridin is a major isoflavone from the rhizome of Belamcanda chinensis (L.) DC., a Chinese medicinal herb of Iridaceae family named as Rhizoma Belamcandae. In Chinese medicine, Rhizoma Belamcandae is commonly used to treat asthma, tonsillitis, and inflammation [16]. Tectoridin has been shown to have estrogenic, anti-oxidative and hepatoprotective effects $[17,18]$, as well as its functions in osteoblastogenesis and osteoclastogenesis [19]. Here, we reported a novel capability of tectoridin in promoting hair growth by activating the Wnt signaling.

\section{Results}

\subsection{Extracts of Rhizoma Belamcandae and Tectoridin Activate Wnt/ $\beta$-Catenin Signalling}

The pTOPFLASH DNA construct containing the TCF-binding consensus sequence for $\beta$-catenin TCF complex was used to quantify the activation of Wnt $/ \beta$-catenin signaling by measuring the driven luciferase activity [20]. In pTOPFLASH-transfected HEK293T cells, the herbal extracts and phytochemicals were applied for $24 \mathrm{~h}$ for activation of luciferase activity. Over 1000 herbal extracts and phytochemicals were screened via the platform. Among over 100 positive hits, the extracts of Rhizoma Belamcandae showed robust activation on the assay of pTOPFLASH DNA construct, and thus which was chosen for further analysis.

Three herbal extracts, i.e., water, 50\% ethanol, and 70\% ethanol, from Rhizoma Belamcandae were tested in pTOPFLASH-transfected HEK293T cells. To control the herbal extracts, HPLC fingerprinting was employed, showing the chemical profile and amount of tectoridin (Figure 1A). The linear regression equation was used to quantify the amount of tectoridin in the extracts. The linear regression data had a good linear relationship with the correlation coefficients $\left(r^{2}\right)$ of tectoridin which was 0.9984 . The linear calibration equation was $\mathrm{Y}=42.26 \mathrm{X}+15.413$, where $\mathrm{Y}$ was the peak area, and $\mathrm{X}$ was the concentration of tectoridin. The content of tectoridin in water extract, $50 \%$ ethanol extract and $70 \%$ ethanol extract of Rhizoma Belamcandae were determined as $0.63 \pm 0.08,1.71 \pm 0.13$, $9.73 \pm 1.34 \mathrm{mg} / \mathrm{g}$, respectively, where $n=4$. 

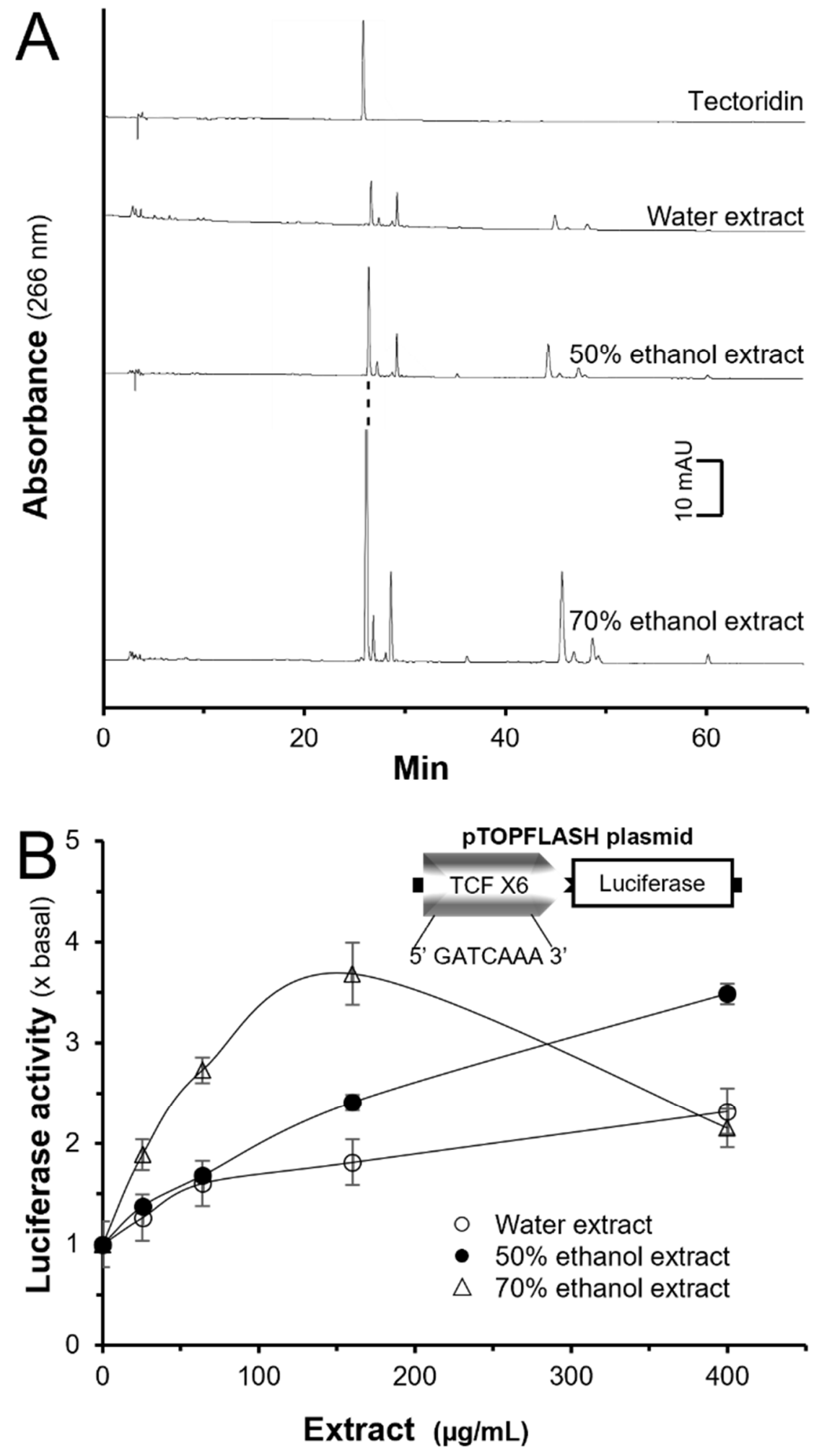

Figure 1. Chemical fingerprints of extracts deriving from Rhizoma Belamcandae and its role in activating Wnt/ $\beta$-catenin signaling. (A): Different extracts of Rhizoma Belamcandae $(10 \mathrm{mg} / \mathrm{mL}$, injection volume $=10 \mu \mathrm{L}$ ) were subjected to HPLC-DAD analysis. The peaks were revealed at $266 \mathrm{~nm}$. Tectoridin at $20 \mathrm{mg} / \mathrm{L}$ was used as the chemical marker here. Representative figures are shown, $n=4$. (B): HEK293T cells were transfected with pTOPFLASH DNA construct (insert) for $4 \mathrm{~h}$. The cultures were treated with different extracts for another $24 \mathrm{~h}$. The cell lysate was subjected for luciferase assays. Data are normalized and expressed as the fold ( $\mathrm{x}$ basal) of control (control group was treated with $0.02 \%$ DMSO), in mean \pm SEM, $n=4$, each with duplicate. 
In dose-dependent manner, the herbal extracts induced the luciferase activity in cultured HEK293T cells having transfection of pTOPFLASH DNA construct (Figure 1B). The luciferase activity, induced by the $70 \%$ ethanol extract, showed the highest induction among the others, having a maximal activation by $\sim 3.5$ folds at $150 \mu \mathrm{g} / \mathrm{mL}$ of the herbal extract. The activation of luciferase was in an order of $70 \%$ ethanol extract $>50 \%$ ethanol extract $>$ water extract.

Rhizoma Belamcandae contains a number of phytochemicals, e.g., isoflavones, xanthone glycosides, stilbenes, simple phenols, and quinones [16-18]. Having AXIN2 as a common drug target of $\mathrm{Wnt} / \beta$-catenin signaling for molecular docking [21], we tested the possible interaction of phytochemicals, i.e., tectoridin, resveratrol, mangiferin, tectorigenin, irigenin, 7-O-methylmangiferin, and irisflorentin; the phytochemicals were identified in Rhizoma Belamcandae [22]. Tectoridin was predicted as the lowest estimated free energy for the binding $(-12.3 \mathrm{KJ} / \mathrm{mol})$, as compared to others (Supplementary Figure S1).

These phytochemicals of Rhizoma Belamcandae, i.e., tectoridin, resveratrol, mangiferin, tectorigenin, irigenin, 7-O-methylmangiferin, and irisflorentin, were applied to pTOPFLASHtransfected HEK293T cells (Supplementary Figure S2). Resveratrol, irigenin, tectorigenin, and tectoridin showed an increase of the luciferase activities. However, tectoridin was the highest content of flavone in the ethanol extracts of Rhizoma Belamcandae, while resveratrol, irigenin, and tectorigenin were close to minimal level (Figure 1A). Besides, the content of tectoridin, Rhizoma Belamcandae was at least few folds higher than the others [22].

Here, we hypothesized that tectoridin could be the active chemical of Rhizoma Belamcandae in triggering the DNA construct. In pTOPFLASH-transfected HEK293T cells, tectoridin was applied in different doses (Figure 2A). Up to $200 \mu \mathrm{M}$ of tectoridin, the cell viability of cultured HEK293T cells did not change (Supplementary Figure S3). Applied tectoridin induced the luciferase activity in a dose-dependent manner at a dose below $20 \mu \mathrm{M}$ : the maximal induction was at $\sim 80 \%$ increase, as compared to the background (Figure 2A). DKK-1, an inhibitor of the Wnt/ $\beta$-catenin signaling by binding to LRP5/ 6 directly to prevent binding of Wnt to its receptors, was used here to test the specificity of tectoridin to the Wnt receptor. The transfected cells were pre-treated with DKK-1 for $1 \mathrm{~h}$ before the application of Wnt3a or tectoridin. The luciferase activity, induced by Wnt3a or tectoridin, was fully inhibited after the pre-treatment with DKK-1 (Figure 2B). The result indicated that tectoridin was acting upstream of Wnt/ $\beta$-catenin signaling or directly binding to the Wnt receptor.

Accumulation of $\beta$-catenin in nucleus is a key step in the Wnt $/ \beta$-catenin signaling pathway. After translocation to the nucleus, $\beta$-catenin binds to T-cell factor (TCF) and activates the transcription of downstream target genes. In transiently transfected HEK293T cells having constitutive expression of $\beta$-catenin-GFP, the effect of tectoridin on $\beta$-catenin subcellular trans-localization was determined (Figure 2C). Valproic acid (VPA) is known to be an inhibitor of GSK-3 $\beta$ [23], serving as a positive control. The localization of $\beta$-cateninGFP was more nuclear-restricted in transfected cells being treated with VPA, or tectoridin, after $24 \mathrm{~h}$, as compared to the control group, suggesting the translocation of $\beta$-catenin under the drug treatment. 


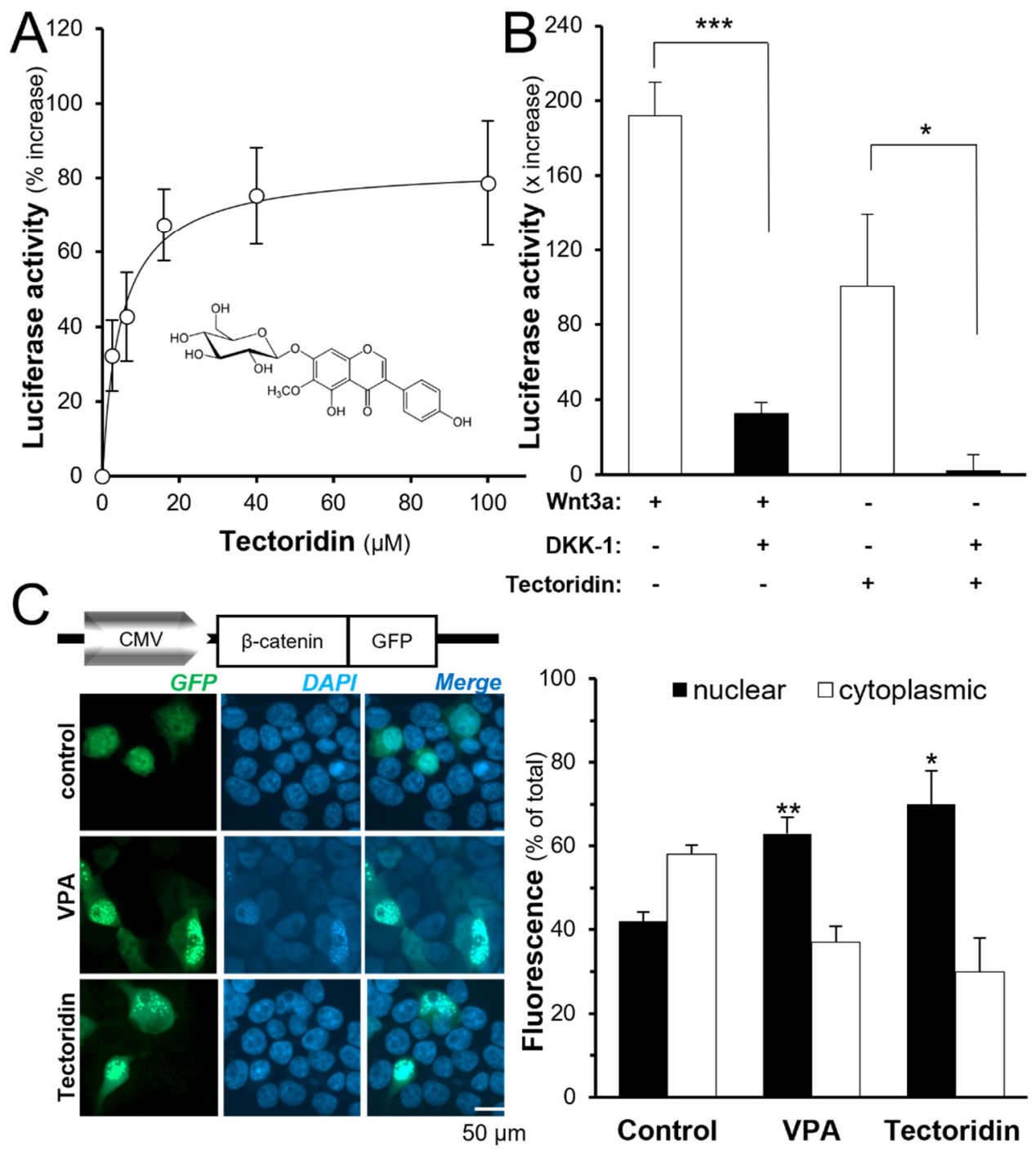

Figure 2. Tectoridin activates Wnt/ $\beta$-catenin signaling. (A) HEK293T cells were transfected with pTOPFLASH DNA construct for $4 \mathrm{~h}$. The cultures were treated with different doses of tectoridin for another $24 \mathrm{~h}$. The cell lysate was used for luciferase assays. (B) HEK293T cells were transfected with as in (A). Then, the cultures were pre-treated with or without DKK-1 (200 ng/mL) for $1 \mathrm{~h}$ before the treatment of tectoridin $(20 \mu \mathrm{M})$, or Wnt3a $(200 \mathrm{ng} / \mathrm{mL})$ for $24 \mathrm{~h}$. (C) HEK293T cells were transfected with a DNA construct containing $\beta$-catenin tagged with green fluorescent protein (GFP; see insert), followed by treatment of tectoridin $(50 \mu \mathrm{M})$ or valproic acid (VPA; an activator of Wnt receptor; $5 \mathrm{mM}$ ) for $24 \mathrm{~h}$. Data are normalized and expressed as the \% of increase, or total, in comparison to control (control group was treated with $0.02 \% \mathrm{DMSO}$ ), in mean $\pm \mathrm{SEM}, n=4$, each with duplicate samples. ${ }^{*} p<0.05,{ }^{* *} p<0.01,{ }^{* * *} p<0.001$.

\subsection{Tectoridin Induces Hair Growth}

DPC is the most common cell model in testing the signaling of hair growth. In cultured DPC, the role of tectoridin was tested in inducing specific gene expressions related to hair growth. The mRNA levels of genes downstream in Wnt signaling pathway, i.e., axin-related protein, (AXIN2), $\beta$-catenin, lymphoid enhancer-binding factor-1 (LEF-1), insulin-like growth factor 1 (IGF-1), and alkaline phosphatase (ALP), were revealed by RTPCR after the treatment of tectoridin for $48 \mathrm{~h}$ in the cultures. The results indicated that the levels of mRNAs encoding AXIN2, LEF-1, and $\beta$-catenin were significantly increased in a dose-dependent manner upon treatments with tectoridin (Figure 3). In parallel, the growth biomarker of DPC, ALP, was also increased by tectoridin significantly. Moreover, the 
expression level of IGF-1, a potent growth factor in promoting hair growth, was increased by over two-folds upon the treatment (Figure 3). Wnt3a served as a positive control here in activating the gene expression.

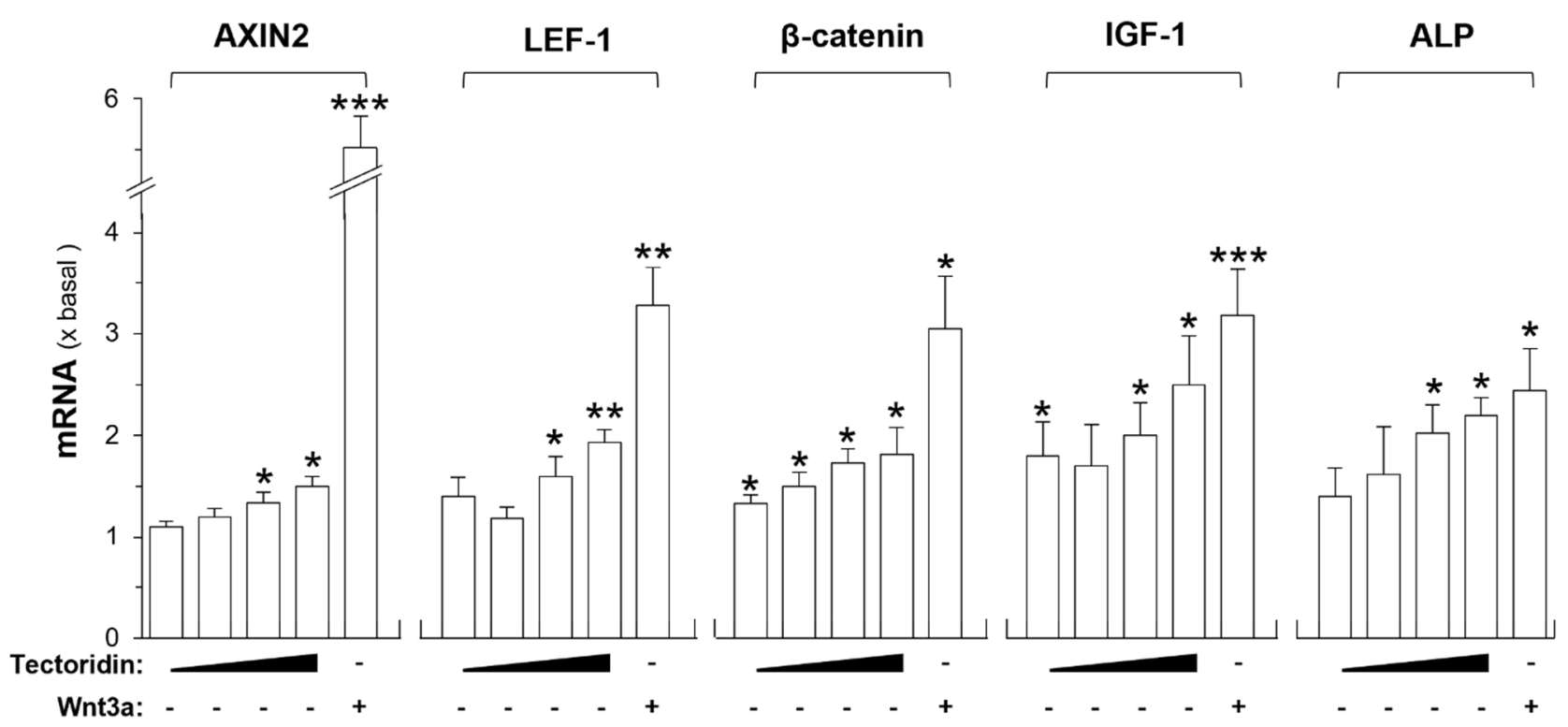

Figure 3. Tectoridin increases expression of downstream target genes of Wnt/ $\beta$-catenin signaling. In cultured DPC, different doses of tectoridin $(3,10,20$ and $50 \mu \mathrm{M}$, as indicated), or Wnt3a (200 ng/mL), were applied for $48 \mathrm{~h}$. The isolated total RNA from the culture was subjected to RT-PCR analyses of AXIN2, LEF-1, $\beta$-catenin, IGF-1, and ALP. Data are normalized and expressed as the fold (x basal) of control (control group was treated with $0.02 \% \mathrm{DMSO}$ ), in mean $\pm \mathrm{SEM}, n=5$, each with duplicate samples. ${ }^{*} p<0.05,{ }^{* *} p<0.01,{ }^{* * *} p<0.001$

Vibrissae hair follicles from mouse is a common ex vivo model in testing hair growth. To evaluate the hair growth promoting property of tectoridin, the isolated vibrissae hair follicles were treated with different doses of tectoridin for $72 \mathrm{~h}$ (Figure 4). The length of hair was measured during the growth, and which was significantly elongated in a dosedependent manner, as compared to control follicle. Under the treatment of 50 or $100 \mu \mathrm{M}$ tectoridin for 3 days, the hair could grow by over $60 \%$ (Figure 4 ): the result was in parallel to that in cell cultures. WAY 316606, an inhibitor of secreted frizzled-related protein-1 (sFRP-1) preventing sFRP-1 interacting with Wnt, served as a positive control showing an induction of hair length by $\sim 25 \%$. 


\section{Day $0 \quad$ Day 3}
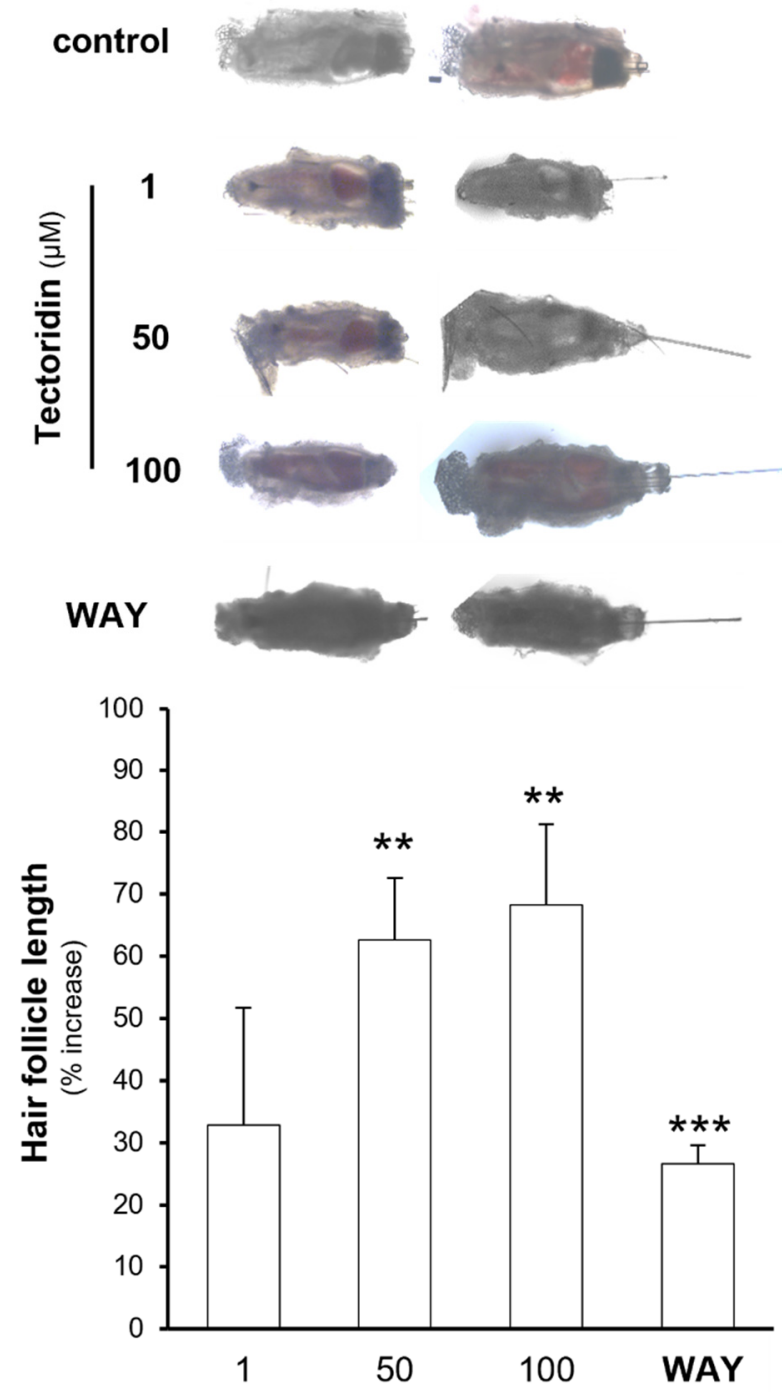

Tectoridin $(\mu \mathrm{M})$

Figure 4. Tectoridin promotes hair shaft elongation. Individual anagen vibrissae hair follicles were isolated from the upper lip pad of 4-week-old C57BL/ 6 male mice and cultured in William's medium $\mathrm{E}$ in $5 \% \mathrm{CO}_{2}$ at $37^{\circ} \mathrm{C}$. The cultures were treated with different doses of tectoridin, or WAY316606 $(2 \mu \mathrm{M})$, as indicated for 3 days. Hair shaft elongation was measured from bottom of hair follicle to the epidermis (upper panel). The measurement of hair length from the hair follicles was performed (low panel). Data are normalized and expressed as the \% of increase in comparison to control (control group was treated with $0.02 \%$ DMSO), in mean \pm SEM, $n=4 .{ }^{* *} p<0.01$, ${ }^{* * *} p<0.001$.

\section{Discussion}

Different forms of hair loss including androgenetic alopecia, senescent alopecia, and telogen effluvium are common in the population over 50 years of age. Currently approved medications, such as Minoxidil and Finasteride, exhibited limited efficacy and unwanted side effects, prompting the search for alternative treatments for hair loss. The hair follicle undergoes cycles of growth, regression, and resting phase throughout life, and the transition of different phases is tightly regulated by DPC, acting as the signaling center of hair growth Besides, Wnt signaling is well-known to play such a role in activating hair growth [20]. The activation of Wnt signaling is initiated by binding of Wnt ligands, including Wnt3a, to Frizzled and LRP5/ 6 co-receptors to stabilize the cytoplasmic $\beta$-catenin by inducing GSK3 $\beta$ phosphorylation. The translocation of $\beta$-catenin into the nucleus activates TCF/LEF 
and downstream target genes. By drug screening platform, we have identified a possible Wnt activator, tectoridin. This notion is supported by its effects in cultures: (i) inducing $\beta$-catenin translocation to nucleus; (ii) activating transcriptions of Wnt signaling-mediated genes and blocked by Wnt receptor inhibitor; and (iii) inducing outgrowth of hair.

This is the first time to identify the hair growth-inducing property of Rhizoma Belamcandae. This medicinal herb has been used as a common traditional Chinese medicine for years. According to historical records, the herb is tailored for various medications, including antipyretic, anti-inflammatory, antidiabetic, anti-angiogenic, and anti-tumor properties [24]. The major phytochemicals in Rhizoma Belamcandae are polyphenols, e.g., isoflavones, xanthone glycosides, stilbenes, simple phenols, and quinones. Indeed, tectoridin is the major isoflavone in Rhizoma Belamcandae, accounting for 4 to $2570 \mathrm{mg} / \mathrm{kg}$ of crude herb [24]. Tectoridin has been proposed to account for the activities of Rhizoma Belamcandae, which has exhibited multiple properties, including anti-microbial and anti-fungal activity, antiinflammatory via inhibition of COX2 activity [25], as well as its estrogenic property [26]. The possible role of tectoridin in alleviating multi-factorial pathogenesis of androgenetic alopecia has been reported. In patients suffering from androgenetic alopecia, inflammation is commonly reported [27]. Administration of COX2 inhibitor has been employed to restore hair growth [28]. Indeed, tectoridin has been demonstrated to possess anti-inflammatory properties, including inhibition of COX2 [25], which therefore could account partly for the hair growth stimulation, besides the action on Wnt signaling. Tectoridin is considered as phytoestrogen having known estrogenic effect: this property may be beneficial in reversing hair loss in females suffering from androgenetic alopecia [29]. Thus, Rhizoma Belamcandae and tectoridin could have a great potential to be developed as hair growth products for androgenetic alopecia, which have the advantage of not having the side effects as the current drugs.

The activation of Wnt signaling by another Chinese herb, roots of Pueraria thomsonii Benth., has been reported in cultured HS68 cell, a foreskin-derived skin fibroblast cell line [30]. Tectoridin, also a significant isoflavone in the roots of $P$. thomsonii, has been proposed to account for this Wnt activation, and which can suppress DKK-1, an inhibitor of Wnt signaling, with regard to the activation of the Wnt signaling. In methanol extract, the tectoridin content in the root of $P$. thomsonii is similar to that of Rhizoma Belamcandae, i.e., $2.97 \mathrm{mg} / \mathrm{g}$ and $2.8 \mathrm{mg} / \mathrm{g}$, respectively [30,31]. In our result, the content of tectoridin in Rhizoma Belamcandae can reach $9.7 \mathrm{mg} / \mathrm{g}$ using 70\% ethanol extraction. Thus, application of extracts deriving from Rhizoma Belamcandae, and/or roots of $P$. thomsonii, in treating androgenetic alopecia could be considered.

\section{Materials and Methods}

\subsection{Materials}

Cell culture media and supplements were purchased from Thermo Fisher Scientific (Waltham, MA, USA), except for those that were specifically indicated. Tectoridin was purchased from Chengdu Herbpurify Ltd. (Chengdu, China) with a purity $\geq 98 \%$. Tectoridin was dissolved in dimethyl sulfoxide (DMSO) to give a stock solution of $50 \mathrm{mM}$. Wnt3a and DKK-1 were purchased from R\&D Systems (Minneapolis, MN, USA). 3-(4,5Dimethylthiazol-2-yl)-2,5-diphenyl tetrazolium bromide (MTT), and valproic acid (VPA) were purchased from Sigma-Aldrich (St. Louis, MO, USA). WAY 316,606 was purchased from ApexBio (Houston, TX, USA).

\subsection{Molecular Docking}

Chemical structures of molecules were downloaded from Pubchem (https:/ / pubchem. ncbi.nlm.nih.gov /, accessed on 2 November 2021); while the structure of AXIN2 was downloaded from Protein Data Bank (PDB, https: / / www.rcsb.org/, accessed on 1 November 2021). Virtual screening was performed on SEESAR software (www.biosolveit.de/LeadIT, accessed on 18 October 2021) following the procedures as below: (i) the binding site was defined according to the residues composing the identified druggable pocket. Ligand 
states, including protonation and tautomeric forms, were automatically assessed in the model using ProToss method, which subsequently generated the most accessible hydrogen positions based on an optimal hydrogen bonding network; (ii) the docking simulation was performed on "Compute LeadIT Docking" mode by using FlexX algorithm. Ten binding conformations for each ligand were generated and (iii) the "assess affinity with HYDE (hydrogen bond and dehydration) in SEESAR" node produced refined binding free energy (i.e., $\Delta G$ ) and estimated HYDE affinity (KiHYDE) for each ligand pose using HYDE rescoring function.

\subsection{Raw Material and HPLC Condition}

The raw material of Rhizoma Belamcandae (rhizome of B. chinensis) was obtained from Guangdong province in 2019 and authenticated by Dr. Tina Dong, one of the authors. The authentication of the herbs was according to Hong Kong Materia Medica Standards. The voucher specimen was deposited at Centre for Chinese medicine at the university. The raw material of Rhizoma Belamcandae was weighed and sonicated with water, $50 \%$ ethanol or $70 \%$ ethanol for $30 \mathrm{~min}$, twice: the volume was 15 times and 10 times, respectively. The herbal extract was dried by a Labconco FreeZone Freeze Dry System. The dried extract was then dissolved in its extraction solution to a final concentration of $100 \mathrm{mg} / \mathrm{mL}$. HPLC-UV chromatographic separation of extracts deriving from Rhizoma Belamcandae was performed on an Agilent HPLC 1200 series system (Agilent, Waldbronn, Germany) equipped with a degasser, a binary pump, an autosampler, a thermos-stated column compartment, and a diode array detector. The herbal extracts were filtered by a $0.22-\mu \mathrm{m}$ filter before separated by an ACE 5 C18 HPLC Column (particle size $5 \mu \mathrm{m}, 4.60 \mathrm{~mm} \times 250 \mathrm{~mm}$ ). The mobile phase was composed of acetonitrile (A) and $0.05 \%$ phosphoric acid in water (B). According to the following gradient program: 0-15 min, linear gradient 18-20\% (A); 15-25 min, linear gradient 20-33\% (A); 25-45 min, linear gradient 33-40\% (A); 45-60 min, linear gradient $40-53 \%$ (A); and 60-65 min, isocratic gradient 53\% (A). A pre-balance period of 5 min was used between each run. The flow rate was set at $1 \mathrm{~mL} / \mathrm{min}$; the column temperature was $25^{\circ} \mathrm{C}$; and the injection volume was $10 \mu \mathrm{L}$. A DAD detector at an absorbance of $266 \mathrm{~nm}$ was used.

\subsection{Cell Culture}

Immortalized DPCs were obtained from Applied Biological Materials (Richmond, BC, Canada). DPC and human embryonic kidney (HEK 293T) cells were cultured in Dulbecco's modified Eagle medium supplemented with 10\% fetal bovine serum (FBS) and 1\% (v/v) penicillin/streptomycin (stock as $10,000 \mathrm{U}$ and $10,000 \mathrm{mg} / \mathrm{mL}$ ) in $5 \% \mathrm{CO}_{2}$ at $37{ }^{\circ} \mathrm{C}$. All culture reagents were purchased from Thermo Fisher Scientific.

\subsection{Animals}

Animals were obtained from Animal and Plant Care Facility of The Hong Kong University of Science and Technology (HKUST) and tested according to the guidelines of Department of Health, The Government of Hong Kong SAR. The experimental procedures were approved by Animal Ethics Committee at the university (reference no.: [20-25] in DH/HT\&A/8/2/2 Pt.1.).

\subsection{Cell Proliferation Assay}

Cell proliferation was assessed by MTT (3-(4,5-dimethylthiazolyl-2)-2,5diphenyltetrazolium bromide) assay. DPC and HEK293T cells were seeded in 96-well plates at a density of 5000 cells per well for $24 \mathrm{~h}$, and then were treated with tectoridin, or extracts from Rhizoma Belamcandae, for $24 \mathrm{~h}$. Afterward, the medium was removed, and $100 \mu \mathrm{L}$ of MTT $(0.5 \mathrm{mg} / \mathrm{mL}$ in complete growth medium) solution was added to each well. Then, cells were incubated at $37^{\circ} \mathrm{C}$ for $1 \mathrm{~h}$. Following the incubation, the medium was replaced with $100 \mu \mathrm{L}$ of DMSO in each well and shaken for 15 min. Absorbance was 
measured using a Multiskan FC Microplate Photometer (Thermo Fisher Scientific) at a wavelength of $570 \mathrm{~nm}$.

\subsection{DNA Transfection and Luciferase Reporter Assay}

The plasmid having TCF/LEF-firefly luciferase reporter (pTOPFLASH) with two repeats, each containing three copies of the TCF-binding site upstream of thymidine kinase minimal promoter, was purchased from Upstate Biotechnology (Lake Placid, NY, USA). Green fluorescent protein (GFP)-tagged $\beta$-catenin construct was a kind gift from Dr. Henderson (University of Sydney, Australia). The reporter construct was then transfected using jetPRIME kit Transfection Reagent (Polyplus transfection, New York, NY, USA) according to the instruction stated on the kit. For the luciferase reporter assay, luciferase assay was performed using PierceTM Firefly Luciferase Glow Assay Kit (Thermo Fisher Scientific Inc., Waltham, MA, USA) according to the instruction stated on the kit. The luminescent reaction was quantified in a GloMax ${ }^{\circledR} 96$ Microplate Luminometer (Thermo Fisher Scientific Inc., Waltham, MA, USA). All values were presented as firefly luciferase activity normalized to the total protein of the samples.

\subsection{Fluorescence Microscopy}

To observe the localization of GFP-tagged $\beta$-catenin, the cells were grown on $10-\mathrm{mm}$ glass coverslips, transfected and incubated as indicated above, then washed twice in PBS, immediately fixed with $4 \%(v / v)$ paraformaldehyde in PBS for $15 \mathrm{~min}$ at room temperature, and subsequently permeabilized with $0.2 \%(v / v)$ Triton X-100 in PBS for 10 min at room temperature. Samples were mounted with ProLong Gold Antifade Mountant with or without DAPI (Thermo Fisher Scientific). Samples were then examined by Zeiss Celldiscoverer 7 automated microscope (Zeiss, Oberkochen, Germany).

\subsection{RT-PCR}

Total RNA was extracted from cultured DPCs using RNAzol ${ }^{\mathrm{TM}}$ Reagent (SigmaAldrich, St. Louis, MO, USA), and $3 \mu \mathrm{g}$ of RNA was reverse transcribed using PrimeScript ${ }^{\mathrm{TM}}$ RT reagent kit (Takara), according to manufacturer's instruction. Template cDNAs were subjected to RT-PCR using the following specific primers: ALP(5'-AGC ACT CCC ACT TCA TCT GG-3' and 5'-TGT CTT CCG AGG AGG TCA AG-3'); LEF-1 (5'-TTC CTT GGT GAA CGA GTC TG-3' and 5'-GTG TTC TCT GGC CTT GTC GT -3'); AXIN2 (5'-GGG AGA AAT GCG TGG ATA C-3' and 5'-CTG CTT GGA GAC AAT GCT GT-3'); IGF-1 (5'-TCA GAA GGG TAG CCC CTA GCT-3' and $5^{\prime}$-TCA AGC CTG GGT ACT TTT AAC CA-3'); $\beta$-catenin (5'-CCC ACT AAT GTC CAG CGT TT-3' and 5'-AAC CAA GCA TTT TCA CCA GG-3'); glyceraldehyde 3-phosphate dehydrogenase (GAPDH) (5'-ACC TGA CCT GCC GTC TAG AA-3' and 5'-TCC ACC ACC CTG TTG CTG TA-3'). Here, GAPDH, a housekeeping gene, was used as an internal control. RT-PCR was performed in LightCycler 480 (Roche Molecular Biochemical, Indianapolis, IN, USA) using KAPA SYBR FAST qPCR kits in accordance with the manufacturer's instruction. The $2^{\Delta \Delta C}$ T method was used to calculate the relative expression levels.

\subsection{Vibrissae Hair Follicle Culture}

Mouse vibrissae hair follicles in anagen phase were carefully isolated from the upper lip pad of 4-week-old C57BL/ 6 male mice. The hair follicles were cultured in Williams E medium (Sigma-Aldrich) supplemented with $1 \%(v / v)$ penicillin/streptomycin solution in $5 \% \mathrm{CO}_{2}$ at $37^{\circ} \mathrm{C}$. Five hair follicles were isolated for each group. Vibrissae hair follicles were incubated in various concentrations of tectoridin or $2 \mu \mathrm{M}$ WAY-316606 (Tocris Bioscience, Ellisville, MO, USA) for 3 days, and increase in the length of hair was measured from days 0 to 3 . 


\subsection{Statistical Analysis and Other Assays}

Protein concentrations were measured by a kit from Bio-Rad (Richmond, CA, USA). Each result was presented as the mean \pm SEM, calculated from 3 to 5 independent samples, with triplicated. Comparisons of the mean for untreated control cells and treated cells were analyzed using one-way analysis of variance (ANOVA) and Student's t test. Significant values were represented as ${ }^{*}, p<0.05,{ }^{* *}, p<0.01,{ }^{* * *}, p<0.001$.

\section{Conclusions}

Tectoridin and extracts of Rhizoma Belamcandae were shown to activate Wnt/ $\beta$ catenin signaling greatly in the assay of pTOPFLASH-transfected cells. Tectoridin was shown to have the capability of activating Wnt/ $\beta$-catenin signaling in human DPC and promoting hair growth in vibrissae hair follicle. Our working hypothesis is the possible intervening of AXIN's role in triggering the Wnt/ $\beta$-catenin signaling by tectoridin (Figure 5). The results suggest a new possible topical medical application of using tectoridin, or extracts of Rhizoma Belamcandae, in treating alopecia. Further work must be carried out in characterizing the multiple pathways of tectoridin/Rhizoma Belamcandae extract acting on the hair follicle and the efficacy in treating androgenetic alopecia.

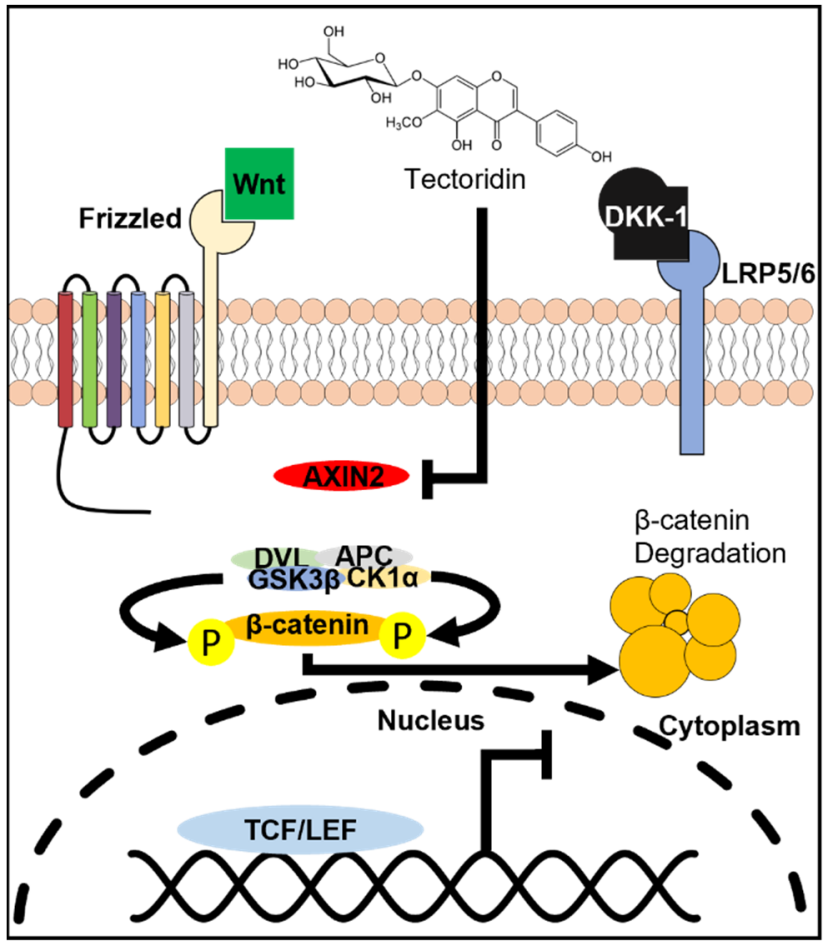

Figure 5. Proposed action of tectoridin in $\mathrm{Wnt} / \beta$-catenin signaling.

Supplementary Materials: The following are available online, Figure S1: Docking image of tectoridin against AXIN2 protein. Figure S2: Luciferase reporter assay of the phytochemicals in Rhizoma Belamcandae. Figure S3: Cytotoxicity of extracts of Rhizoma Belamcandae and tectoridin in cultures.

Author Contributions: Conceptualization, G.K.-W.Y.; data curation, G.K.-W.Y. and B.S.-Y.H.; software, L.S.-Y.L.; project administration, K.W.-K.T.; supervision, K.W.-K.T.; validation, T.T.-X.D.; writing-original draft, G.K.-W.Y.; writing—review and editing, K.W.-K.T. All authors have read and agreed to the published version of the manuscript. 
Funding: This research was funded by the Key-Area Research and Development Program of Guangdong Province (2020B1111110006); Special project of Foshan University of science and technology in 2019 (FSUST19-SRI10); GBA Institute of Collaborate Innovation (GICI-022); Shenzhen Science and Technology Innovation Committee (ZDSYS201707281432317; JCYJ20170413173747440; JCYJ2018030 6174903174), Zhongshan Municipal Bureau of Science and Technology (ZSST20SC03); Guangzhou Science and Technology Committee Research Grant (GZSTI16SC02; GZSTI17SC02); Hong Kong RGC Theme-based Research Scheme (T13-605/18-W); Hong Kong Innovation Technology Fund (UIM/340, UIM/385, ITS/500/18FP; ITCPD/17-9; TUYF19SC02, PD18SC01 and HMRF18SC06; HMRF20SC07; AFD20SC01).

Institutional Review Board Statement: The study was conducted according to the guidelines of the Animals (Control of Experiments) Ordinance (Cap. 340) and approved by the Committee on Research Practice and APCF at HKUST, and Department of Health, Hong Kong. ((20-25) in DH/HT\&A/8/2/2 Pt.1 and 23 March 2020).

Informed Consent Statement: Not applicable.

Data Availability Statement: The data presented in this study are available upon request from the corresponding author.

Conflicts of Interest: All authors declare that they have no conflict of interest.

Sample Availability: Not applicable.

\section{References}

1. Cotsarelis, G.; Millar, S.E. Towards a molecular understanding of hair loss and its treatment. Trends Mol. Med. 2001, 7, 293-301. [CrossRef]

2. Paus, R.; Cotsarelis, G. The biology of hair follicles. N. Engl. J. Med. 1999, 341, 491-497. [CrossRef]

3. Huelsken, J.; Vogel, R.; Erdmann, B.; Cotsarelis, G.; Birchmeier, W. $\beta$-catenin controls hair follicle morphogenesis and stem cell differentiation in the skin. Cell 2001, 105, 533-545. [CrossRef]

4. Kishimoto, J.; Burgeson, R.E.; Morgan, B.A. Wnt signalling maintains the hair-inducing activity of the dermal papilla. Genes Dev. 2000, 14, 1181-1185. [CrossRef]

5. Botchkarev, V.A.; Kishimoto, J. Molecular control of epithelial-mesenchymal interactions during hair follicle cycling. J. Investig. Dermatol. Symp. Proc. 2003, 8, 46-55. [CrossRef] [PubMed]

6. Yang, C.C.; Cotsarelis, G. Review of hair follicle dermal cells. J. Dermatol. Sci. 2010, 57, 2-11. [CrossRef] [PubMed]

7. Morgan, B.A. The dermal papilla: An instructive niche for epithelial stem and progenitor cells in development and regeneration of the hair follicle. Cold Spring Harb. Perspect. Biol. 2014, 4, a015180. [CrossRef] [PubMed]

8. Qi, J.; Garza, L.A. An overview of alopecias. Cold Spring Harb. Perspect. Med. 2014, 4, a013615. [CrossRef] [PubMed]

9. Gordon, K.A.; Tosti, A. Alopecia: Evaluation and treatment. Clin. Cosmet. Investig. Dermatol. 2011, 4, 101-106. [CrossRef] [PubMed]

10. Cardoso, C.O.; Tolentino, S.; Gratieri, T.; Cunha-Filho, M.; Lopez, R.F.; Gelfuso, G.M. Topical treatment for scarring and non-scarring alopecia: An overview of the current evidence. Clin. Cosmet. Investig. Dermatol. 2021, 14, 485-499. [CrossRef]

11. Collins, C.A.; Kretzschmar, K.; Watt, F.M. Reprogramming adult dermis to a neonatal state through epidermal activation of $\beta$-catenin. Development 2011, 138, 5189-5199. [CrossRef] [PubMed]

12. Rajendran, R.L.; Gangadaran, P.; Seo, C.H.; Kwack, M.H.; Oh, J.M.; Lee, H.W.; Gopal, A.; Sung, Y.K.; Jeong, S.Y.; Lee, S.-W.; et al. Macrophage-derived extracellular vesicle promotes hair growth. Cells 2020, 9, 856. [CrossRef]

13. Li, Y.H.; Zhang, K.; Ye, J.X.; Lian, X.H.; Yang, T. Wnt10b promotes growth of hair follicles via a canonical Wnt signalling pathway. Clin. Exp. Dermatol. 2011, 36, 534-540. [CrossRef] [PubMed]

14. Shin, S.H.; Bak, S.-S.; Kim, M.K.; Sung, Y.K.; Kim, J.C. Baicalin, a flavonoid, affects the activity of human dermal papilla cells and promotes anagen induction in mice. Naunyn Schmiedebergs Arch. Pharmacol. 2015, 388, 583-586. [CrossRef]

15. Yazici, Y.; Smith, S.; Swearingen, C.; Simsek, I.; DiFrancesco, A.; Hood, J. Safety and efficacy of a topical treatment (SM04554) for androgenetic alopecia (AGA): Results from a phase 1 Trial: 3299. J. Am. Acad. Dermatol. 2016, 74, AB138.

16. Zhang, L.; Wei, K.; Xu, J.; Yang, D.; Zhang, C.; Wang, Z.; Li, M. Belamcanda chinensis (L.) DC-an ethnopharmacological, phytochemical and pharmacological review. J. Ethnopharmacol. 2016, 186, 1-13. [CrossRef] [PubMed]

17. Lee, H.U.; Bae, E.A.; Kim, D.H. Hepatoprotective effect of tectoridin and tectorigenin on tert-butyl hyperoxide-induced liver injury. J. Pharmacol. Sci. 2005, 97, 541-544. [CrossRef] [PubMed]

18. Han, T.; Cheng, G.; Liu, Y.; Yang, H.; Hu, Y.T.; Huang, W. In vitro evaluation of tectoridin, tectorigenin and tectorigenin sodium sulfonate on antioxidant properties. Food Chem. Toxicol. 2012, 50, 409-414. [CrossRef]

19. Wang, J.; Tang, Y.; Lv, X.; Zhang, J.; Ma, B.; Wen, X.; Bao, Y.; Wang, G. Tectoridin inhibits osteoclastogenesis and bone loss in a murine model of ovariectomy-induced osteoporosis. Exp. Gerontol. 2020, 140, 111057. [CrossRef] 
20. Millar, S.E.; Willert, K.; Salinas, P.C.; Roelink, H.; Nusse, R.; Sussman, D.J.; Barsh, G.S. WNT signalling in the control of hair growth and structure. Dev. Biol. 1999, 207, 133-149. [CrossRef]

21. Chen, D.; Jing, C.; Cai, J.; Wu, J.; Wang, S.; Yin, J.; Li, X.; Li, L.; Hao, X. Design, synthesis, and structural optimization of lycorine-derived phenanthridine derivatives as Wnt/ $\beta$-catenin signaling pathway agonists. J. Nat. Prod. 2015, 79, 180-188. [CrossRef]

22. Li, J.; Li, W.; Huang, W.; Cheung, A.W.H.; Bi, C.W.C.; Duan, R.; Guo, A.J.Y.; Dong, T.T.X.; Tsim, K.W.K. Quality evaluation of Rhizoma Belamcandae (Belamcanda chinensis (L.) DC.) by using high-performance liquid chromatography coupled with diode array detector and mass spectrometry. J. Chromatogr. A 2009, 1216, 2071-2078. [CrossRef] [PubMed]

23. Jo, S.J.; Choi, S.J.; Yoon, S.Y.; Lee, J.Y.; Park, W.; Park, P.; Kim, K.H.; Eun, H.C.; Kwon, O. Valproic acid promotes human hair growth in in vitro culture model. J. Dermatol. Sci. 2013, 72, 16-24. [CrossRef] [PubMed]

24. Woźniak, D.; Matkowski, A. Belamcandae chinensis rhizome-A review of phytochemistry and bioactivity. Fitoterapia 2015, 107, 1-14. [CrossRef] [PubMed]

25. Kim, Y.P.; Yamada, M.; Lim, S.S.; Lee, S.H.; Ryu, N.; Shin, K.H.; Ohuchi, K. Inhibition by tectorigenin and tectoridin of prostaglandin E2 production and cyclooxygenase-2 induction in rat peritoneal macrophages. Biochim. Biophys. Acta Biomembr. 1999, 1438, 399-407. [CrossRef]

26. Shin, J.-E.; Bae, E.-A.; Lee, Y.C.; Ma, J.Y.; Kim, D.H. Estrogenic effect of main components kakkalide and tectoridin of Puerariae Flos and their metabolites. Biol. Pharm. Bull. 2006, 29, 1202-1206. [CrossRef]

27. Peyravian, N.; Deo, S.; Daunert, S.; Jimenez, J.J. The inflammatory aspect of male and female pattern hair loss. J. Inflamm. Res. 2020, 13, 879-881. [CrossRef]

28. Kang, J.I.; Kim, S.C.; Kim, M.K.; Boo, H.-J.; Kim, E.-J.; Im, G.-J.; Kim, Y.H.; Hyun, J.-W.; Kang, J.-H.; Koh, Y.-S.; et al. Effects of dihydrotestosterone on rat dermal papilla cells in vitro. Eur. J. Pharmacol. 2015, 757, 74-83. [CrossRef]

29. Ohnemus, U.; Uenalan, M.; Inzunza, J.; Gustafsson, J.A.; Paus, R. The hair follicle as an estrogen target and source. Endocr. Rev. 2006, 27, 677-706. [CrossRef]

30. Ahn, Y.J.; Chang, Y.H.; Lee, S.Y.; Jin, M.H. A study on the whitening effects of Pueraria thomsonii extract and its three tectorigenin derivatives. J. Soc. Cosmet. Sci. Korea 2019, 45, 49-56.

31. Xu, L.-L.; Zhang, Y.; Chai, Y.; Kuan, C.; Hai-Dong, W.; Chun-Guo, Y.; Min, Y.; Xue, Q. Differentiation of Belamcandae Rhizoma and Iridis Tectori Rhizoma by thin-layer chromatography and high-performance liquid chromatography. World J. Tradit. Chin. Med. 2021, 7, 63-70. 